\title{
The Stein-Weiss theorem for the ergodic Hilbert transform
}

\author{
by \\ LASHA EPHREMIDZE (Tbilisi)
}

\begin{abstract}
The Stein-Weiss theorem that the distribution function of the Hilbert transform of the characteristic function of $E$ depends only on the measure of $E$ is generalized for the ergodic Hilbert transform in the case of a one-parameter flow of measurepreserving transformations on a $\sigma$-finite measure space.
\end{abstract}

1. Introduction. Let $\left(T_{t}\right)_{t \in \mathbb{R}}$ be an ergodic group of measure-preserving transformations on a $\sigma$-finite measure space $(X, \mathbb{S}, \mu)$. The ergodic Hilbert transform of an integrable function $f \in L(X)$ is defined by

$$
\mathbb{H}(f)(x)=\lim _{\delta \rightarrow 0+} \frac{1}{\pi} \int_{\{\delta \leq|t| \leq 1 / \delta\}} \frac{f\left(T_{-t} x\right)}{t} d t .
$$

The limit in (1) exists and consequently $\mathbb{H}(f)(x)$ is well defined for a.a. $x \in X$ (see [6], [7]).

If $T_{t}, t \in \mathbb{R}$, are the translations on the real line, $x \mapsto x+t$, and $f \in L(\mathbb{R})$, then we get the usual Hilbert transform, $\mathbb{H}(f)=H(f)$, while if $T_{t}, t \in \mathbb{R}$, are the rotations of the unit circle $\mathbb{T}, e^{i \theta} \mapsto e^{i(\theta+t)}$, and $f \in L(\mathbb{T})$, then we get the conjugate, $\mathbb{H}(f)=\widetilde{f}$ (see [2], [3]).

The two theorems below which identify the distribution functions of the Hilbert transform and of the conjugate of $\mathbb{1}_{E}$, the characteristic function of a measurable set $E$, are well known and belong to Stein and Weiss [8].

Theorem A. Let $E \subset \mathbb{R}$ be a measurable set with finite Lebesgue measure $m(E)$. Then

$$
\begin{aligned}
m\left\{x \in \mathbb{R}: H\left(\mathbb{1}_{E}\right)(x)>\lambda\right\} & =m\left\{x \in \mathbb{R}: H\left(\mathbb{1}_{E}\right)(x)<-\lambda\right\} \\
& =\Psi(m(E), \lambda), \quad \lambda \geq 0 .
\end{aligned}
$$

2000 Mathematics Subject Classification: 28D10, 42A50.

Key words and phrases: ergodic Hilbert transform, Stein-Weiss theorem. 
Theorem B. Let $E \subset \mathbb{T}$ be a measurable set. Then $m\left\{x \in \mathbb{T}: \widetilde{\mathbb{1}}_{E}(x)>\lambda\right\}=m\left\{x \in \mathbb{T}: \widetilde{\mathbb{1}}_{E}(x)<-\lambda\right\}=\Phi(m(E), \lambda), \quad \lambda \geq 0$.

The original proof of the authors uses real methods, while there exist shorter proofs applying complex methods ([1], [4]). The exact form of the functions $\Psi$ and $\Phi$ is also well known (see [8], [9]):

$$
\Psi(\xi, \lambda)=\frac{\xi}{\sinh (\pi \lambda)}, \quad \Phi(\xi, \lambda)=2 \arctan \frac{\sin (\xi / 2)}{\sinh (\pi \lambda)} .
$$

In [3] an analogue of Theorem B is proved for the ergodic Hilbert transform.

TheOREM $\mathrm{B}^{\prime}$. Let $\left(T_{t}\right)_{t \in \mathbb{R}}$ be an ergodic group of measure-preserving transformations on a finite measure space $(X, \mathbb{S}, \mu)$ with $\mu(X)=2 \pi$ and let $E \in \mathbb{S}$. Then

$$
\begin{aligned}
\mu\left\{x \in X: \mathbb{H}\left(\mathbb{1}_{E}\right)(x)>\lambda\right\} & =\mu\left\{x \in X: \mathbb{H}\left(\mathbb{1}_{E}\right)(x)<-\lambda\right\} \\
& =\Phi(\mu(E), \lambda), \quad \lambda \geq 0 .
\end{aligned}
$$

The methods developed in [3], as emphasized there, do not allow us to generalize Theorem A. In this paper we resort to a more refined technique to achieve this goal. As a result, the generalization of the Stein-Weiss theorem is obtained for infinite measure spaces.

TheOREM. Let $\left(T_{t}\right)_{t \in \mathbb{R}}$ be an ergodic group of measure-preserving transformations on a $\sigma$-finite measure space $(X, \mathbb{S}, \mu)$ with $\mu(X)=\infty$ and let $E \in \mathbb{S}$ with $\mu(E)<\infty$. Then

$$
\begin{aligned}
\mu\left\{x \in X: \mathbb{H}\left(\mathbb{1}_{E}\right)(x)>\lambda\right\} & =\Psi(\mu(E), \lambda) \\
& =\mu\left\{x \in X: \mathbb{H}\left(\mathbb{1}_{E}\right)(x)<-\lambda\right\}, \quad \lambda \geq 0 .
\end{aligned}
$$

In what follows we always assume that $\mu(X)=\infty$.

1. Some known facts. The classical maximal Hilbert transform

$$
H^{*}(h)(t)=\sup _{\delta>0} \frac{1}{\pi} \int_{\{\delta \leq|\tau|\}} \frac{h(t-\tau)}{\tau} d \tau, \quad h \in L(\mathbb{R}),
$$

is of weak type $(1,1)$, i.e.

$$
m\left\{t \in \mathbb{R}: H^{*}(h)(t)>\lambda\right\} \leq \frac{C}{\lambda}\|h\|_{L(\mathbb{R})}, \quad \lambda>0
$$

(see [7, Theorem 3.5.3]). Consequently,

$$
m\left\{t \in \mathbb{R}: H^{\Delta}(h)(t)>\lambda\right\} \leq \frac{2 C}{\lambda}\|h\|_{L(\mathbb{R})}, \quad \lambda>0,
$$


where

$$
H^{\Delta}(h)=\sup _{0<\delta<\delta^{\prime}} \frac{1}{\pi} \int_{\left\{\delta \leq|\tau| \leq \delta^{\prime}\right\}} \frac{h(t-\tau)}{\tau} d \tau .
$$

The proof of Theorem 3.6.1 in [7] (which states that the maximal ergodic Hilbert transform

$$
\mathbb{H}^{*}(f)(x)=\sup _{\delta>0} \frac{1}{\pi} \int_{\{\delta \leq|t| \leq 1 / \delta\}} \frac{f\left(T_{-t} x\right)}{t} d t, \quad f \in L(X),
$$

is of weak type $(1,1)$ ) goes over without any change for $\sigma$-finite measure spaces, and if we apply the inequality (4) instead of (3) in this proof, then we infer that the operator

$$
\mathbb{H}^{\Delta}(f)(x)=\sup _{0<\delta<\delta^{\prime}} \frac{1}{\pi} \int_{\left\{\delta \leq|t| \leq \delta^{\prime}\right\}} \frac{f\left(T_{-t} x\right)}{t} d t
$$

is of weak type $(1,1)$. Consequently,

$$
\mu\left\{x \in X: \mathbb{H}^{\Delta}(f)(x)>\lambda\right\}<\infty
$$

for each $f \in L(X)$ and $\lambda>0$.

The existence of the limit in (1) actually states that

$$
\mathbb{H}^{a}(f)(x)=\lim _{\delta \rightarrow 0+} \frac{1}{\pi} \int_{\{a \leq|t| \leq 1 / \delta\}} \frac{f\left(T_{-t} x\right)}{t} d t, \quad a>0,
$$

exists for a.a. $x \in X$ since the existence of

$$
\mathbb{H}_{a}(f)(x)=\lim _{\delta \rightarrow 0+} \frac{1}{\pi} \int_{\{\delta \leq|t| \leq a\}} \frac{f\left(T_{-t} x\right)}{t} d t
$$

for a.a. $x \in X$ can be obtained by standard arguments when we know that the usual Hilbert transform of an integrable function exists on the real line. Hence

$$
\int_{\left\{\delta \leq|t| \leq \delta^{\prime}\right\}} \frac{f\left(T_{-t} x\right)}{t} d t \rightarrow 0 \quad \text { as } \delta, \delta^{\prime} \rightarrow \infty
$$

and consequently,

$$
\mathbb{H}_{a}^{\Delta}(f)(x)=\sup _{\delta>a}\left|\mathbb{H}_{\delta}(f)(x)-\mathbb{H}(f)(x)\right|
$$

monotonically converges to 0 for a.a. $x \in X$ as $a \rightarrow \infty$. Moreover, taking into account (5), we have the convergence in measure

$$
\mathbb{H}_{a}^{\Delta}(f) \rightrightarrows 0 \quad \text { as } a \rightarrow \infty .
$$


A group $\left(T_{t}\right)_{t \in \mathbb{R}}$ of measure preserving transformations is called conservative ergodic if $0 \leq f \in L(X), f \not \equiv 0$ implies

$$
\lim _{a \rightarrow \infty} \int_{0}^{a} f\left(T_{t} x\right) d t=\infty \quad \text { for a.a. } x \in X .
$$

If an ergodic group on a $\sigma$-finite measure space is not isomorphic to the group of translations on the real line, then it is conservative ergodic. Thus, without loss of generality, we can assume in our proof that $\left(T_{t}\right)_{t \in \mathbb{R}}$ is conservative ergodic.

Fix any non-negative function $g$ on $X$ satisfying

$$
\int_{X} g d \mu=1 .
$$

The continuous version of the Chacon-Ornstein Theorem (see [5]) states that, for every $f \in L(X)$,

$$
\lim _{a \rightarrow \infty} \frac{\int_{0}^{a} f\left(T_{t} x\right) d t}{\int_{0}^{a} g\left(T_{t} x\right) d t}=\int_{X} f d \mu \quad \text { for a.a. } x \in X .
$$

Thus, if $\gamma_{x}: a \mapsto \gamma_{x}(a), x \in X$, is the system of functions of variable $a$ defined by

$$
\gamma_{x}(a)=\int_{0}^{a} g\left(T_{t} x\right) d t
$$

(the standard application of Fubini's theorem implies that, for a.a. $x \in X$, the function $\gamma_{x}$ is well defined for each $a \geq 0$ ), then, for each measurable $A \in \mathbb{S}$, the equality

$$
\lim _{a \rightarrow \infty} \frac{1}{\gamma_{x}(a)} \int_{0}^{a} \mathbb{1}_{A}\left(T_{t} x\right) d t=\int_{X} \mathbb{1}_{A} d \mu,
$$

i.e.

$$
\lim _{a \rightarrow \infty} \frac{m\left\{t \in(0, a): T_{t} x \in A\right\}}{\gamma_{x}(a)}=\mu(A)
$$

holds for a.a. $x \in X$. In addition, if $\mu(A)<\infty$, then the ergodic theorem for infinite measure spaces (see [7, Remark 2.2.4]) states that

$$
\lim _{a \rightarrow \infty} \frac{1}{a} \int_{0}^{a} \mathbb{1}_{A}\left(T_{t} x\right) d t=0 .
$$

Note also that $\gamma_{x}$ is a non-increasing continuous function for a.a. $x \in X$ and since $\left(T_{t}\right)_{t \in \mathbb{R}}$ is conservative ergodic (see $(8)$ ), we have

$$
\lim _{a \rightarrow \infty} \gamma_{x}(a)=\infty
$$


3. Auxiliary lemmas. The following lemma is well known for probability spaces in the case where the sequence $\left\{G_{n}\right\}_{n=1}^{\infty}$ consists of one function. Its proof is given for the sake of completeness to show that the infinite measure is no obstacle.

LEMMA 1. Let $\left\{F_{n}\right\}_{n=1}^{\infty},\left\{G_{n}\right\}_{n=1}^{\infty}$ be sequences of measurable functions on a $\sigma$-finite measure space such that the distribution functions of $G_{n}$ are convergent to some function $\psi, \lim _{n \rightarrow \infty} \mu\left\{G_{n}>\lambda\right\}=\psi(\lambda)$ for each $\lambda \in \mathbb{R}$, and $F_{n}-G_{n} \rightrightarrows 0$. Then

$$
\lim _{n \rightarrow \infty} \mu\left\{F_{n}>\lambda\right\}=\psi(\lambda)
$$

for every point $\lambda$ of continuity of $\psi$.

Proof. We have

$$
\begin{aligned}
\left\{G_{n}>\lambda+\varepsilon\right\} \backslash\left\{F_{n}-G_{n} \leq-\varepsilon\right\} & \subset\left\{F_{n}>\lambda\right\} \\
& \subset\left\{G_{n}>\lambda-\varepsilon\right\} \cup\left\{F_{n}-G_{n} \geq \varepsilon\right\} .
\end{aligned}
$$

Therefore

$$
\begin{aligned}
\mu\left\{G_{n}>\lambda+\varepsilon\right\}-\mu\left\{F_{n}-G_{n} \leq-\varepsilon\right\} & \leq \mu\left\{F_{n}>\lambda\right\} \\
& \leq \mu\left\{G_{n}>\lambda-\varepsilon\right\}+\mu\left\{F_{n}-G_{n} \geq \varepsilon\right\}
\end{aligned}
$$

for each $\varepsilon>0$ and positive integer $n$. Since $\lim _{n \rightarrow \infty} \mu\left\{\left|F_{n}-G_{n}\right|>\varepsilon\right\}=0$, we get

$$
\begin{aligned}
\psi(\lambda+\varepsilon) & =\lim _{n \rightarrow \infty} \mu\left\{G_{n}>\lambda+\varepsilon\right\} \leq \liminf _{n \rightarrow \infty} \mu\left\{F_{n}>\lambda\right\} \\
& \leq \limsup _{n \rightarrow \infty} \mu\left\{F_{n}>\lambda\right\} \leq \lim _{n \rightarrow \infty} \mu\left\{G_{n}>\lambda-\varepsilon\right\}=\psi(\lambda-\varepsilon)
\end{aligned}
$$

and (14) follows from the fact that $\lambda$ is a continuity point of $\psi$.

If $h$ is a locally integrable function on $\mathbb{R}$ and $a>0$, then let

$$
H_{(0, a)}(h)(t):=\lim _{\delta \rightarrow 0+} \frac{1}{\pi} \int_{\{\delta \leq|\tau| \leq \min (t, a-t)\}} \frac{h(t-\tau)}{\tau} d \tau, \quad 0<t<a .
$$

We assume that $H_{(0, a)}(h)$ is equal to 0 outside the interval $(0, a)$, and thus the value of the operator $H_{(0, a)}$ at $h$ depends only on the values of $h$ in $(0, a), H_{(0, a)}(h)=H_{(0, a)}\left(\mathbb{1}_{(0, a)} h\right)$.

Observe that if $D_{\kappa}, \kappa>0$, denotes the dilation operator,

$$
D_{\kappa} h(t)=h(\kappa t),
$$

then a simple change of variables under the integration gives $H_{(0, a)}(h)(t)=$ $H_{(0, a / \kappa)}\left(D_{\kappa} h\right)(t / \kappa)$, which implies

$$
\frac{1}{\kappa} m\left\{H_{(0, a)}(h)>\lambda\right\}=m\left\{H_{(0, a / \kappa)}\left(D_{\kappa} h\right)>\lambda\right\}, \quad \lambda>0 .
$$


For $f \in L(X)$, let $f_{x}(t)=f\left(T_{t} x\right)$. For a.a. $x \in X$, the function $f_{x}$ is locally integrable and in that case we have (see (6))

$$
H_{(0, a)}\left(f_{x}\right)(t)=\mathbb{H}_{\min (t, a-t)} f\left(T_{t} x\right), \quad 0<t<a .
$$

LEMma 2. Let $\left(T_{t}\right)_{t \in \mathbb{R}}$ be a conservative ergodic flow of measure-preserving transformations on a $\sigma$-finite measure space $(X, \mathbb{S}, \mu)$ and let functions $\left(\gamma_{x}\right)_{x \in X}$ be defined by (10). If $f \in L(X)$ and $\lambda>0$ is a continuity point of the distribution function of $\mathbb{H}(f)$, then

$$
\begin{aligned}
\lim _{a \rightarrow \infty} \frac{m\left\{H_{(0, a)}\left(f_{x}\right)>\lambda\right\}}{\gamma_{x}(a)} & =\lim _{a \rightarrow \infty} \frac{m\left\{0<t<a: H_{(0, a)}\left(f_{x}\right)(t)>\lambda\right\}}{\gamma_{x}(a)} \\
& =\mu\{\mathbb{H}(f)>\lambda\}
\end{aligned}
$$

for a.a. $x \in X$.

Proof. Suppose (18) does not hold and there exists $\varepsilon>0$ such that

$$
\begin{aligned}
& \mu\left\{x \in X: \limsup _{a \rightarrow \infty}\left(\frac{m\left\{0<t<a: H_{(0, a)}\left(f_{x}\right)(t)>\lambda\right\}}{\gamma_{x}(a)}\right.\right. \\
&-\mu\{\mathbb{H}(f)>\lambda\})>\varepsilon\}>0 .
\end{aligned}
$$

It follows that for some $\delta>0$,

$$
\begin{aligned}
& \mu\left\{x \in X: \limsup _{a \rightarrow \infty}\left(\frac{m\left\{0<t<a: H_{(0, a)}\left(f_{x}\right)(t)>\lambda\right\}}{\gamma_{x}(a)}\right.\right. \\
&-\mu\{\mathbb{H}(f)>\lambda-\delta\})>\varepsilon\}>0 .
\end{aligned}
$$

Hence, taking into account (11) for the measurable set $\{x \in X: \mathbb{H}(f)(x)>$ $\lambda-\delta\}$, we infer that

$$
\begin{gathered}
m\left\{0<t<a: H_{(0, a)}\left(f_{x}\right)(t)>\lambda\right\} \\
\limsup _{a \rightarrow \infty} \frac{-m\left\{0<t<a: \mathbb{H}(f)\left(T_{t} x\right)>\lambda-\delta\right\}}{\gamma_{x}(a)}>\varepsilon
\end{gathered}
$$

for every $x$ from a set of positive measure.

Because of (7), we can take $a_{0}$ so large that

$$
\mu(A)<\varepsilon,
$$

where $A=\left\{x \in X: \mathbb{H}_{a_{0}}^{\Delta}(f)(x)>\delta\right\}$.

Fix any $x$ for which (11), (13) and (19) hold. If $a$ is large enough and $t$ is such that $\min (t, a-t)>a_{0}$ and $T_{t} x \notin A$, then

$\left|\mathbb{H}_{\min (t, a-t)}(f)\left(T_{t} x\right)-\mathbb{H}(f)\left(T_{t} x\right)\right| \leq \mathbb{H}_{\min (t, a-t)}^{\Delta}(f)\left(T_{t} x\right) \leq \mathbb{H}_{a_{0}}^{\Delta}(f)\left(T_{t} x\right) \leq \delta$, and consequently (see (17)),

$$
H_{(0, a)}\left(f_{x}\right)(t)>\lambda \Rightarrow \mathbb{H}(f)\left(T_{t} x\right)>\lambda-\delta .
$$


Hence the left hand side of (19) is not greater than

$$
\limsup _{a \rightarrow \infty} \frac{m\left(0, a_{0}\right)+m\left(a-a_{0}, a\right)+m\left\{t \in(0, a): T_{t} x \in A\right\}}{\gamma_{x}(a)},
$$

which is equal to $\mu(A)$ (see (13), (11)). This contradicts (19) because of (20).

If there exists $\varepsilon>0$ such that

$$
\mu\left\{x \in X: \limsup _{a \rightarrow \infty}\left(\frac{m\left\{0<t<a: H_{(0, a)}\left(f_{x}\right)(t)>\lambda\right\}}{\gamma_{x}(a)}-\mu\{\mathbb{H}(f)>\lambda\}\right)<-\varepsilon\right\}>0,
$$

then $\mu\{\mathbb{H}(f)>\lambda\}$ can be replaced by $\mu\{\mathbb{H}(f)>\lambda+\delta\}$ for some $\delta>0$ and the implication $\mathbb{H}(f)\left(T_{t} x\right)>\lambda+\delta \Rightarrow H_{(0, a)}\left(f_{x}\right)(t)>\lambda$ should be used instead of (21). The rest of the proof is the same.

Lemma 3. Let $h$ be a non-negative integrable function on $\mathbb{R}$ which vanishes outside the interval $(0, a), a>0$. Then

$$
\left|H_{(0, a)}(h)(t)-H(h)(t)\right| \leq \begin{cases}-3 H(h)(-t) & \text { for } 0<t \leq a / 2, \\ 3 H(h)(2 a-t) & \text { for } a / 2 \leq t<a .\end{cases}
$$

Proof. If $0<t \leq a / 2$ and $2 t \leq \tau \leq a$, then $\tau+t \leq 3(\tau-t)$ and

$$
\begin{aligned}
0 \leq H_{(0, a)}(h)(t)-H(h)(t) & =\frac{1}{\pi} \int_{2 t}^{a} \frac{h(\tau)}{\tau-t} d \tau \leq \frac{3}{\pi} \int_{2 t}^{a} \frac{h(\tau)}{\tau+t} d \tau \\
& \leq \frac{3}{\pi} \int_{0}^{a} \frac{h(\tau)}{\tau+t} d \tau=-3 H(h)(-t) .
\end{aligned}
$$

To prove the second inequality, we apply the first for $h_{0}(t)=h(a-t)$.

Lemma 4. Let $\mathcal{E} \subset \mathbb{R}_{+}$be such that

$$
\lim _{a \rightarrow \infty} \frac{m(\mathcal{E} \cap[0, a))}{a}=0
$$

and let $\gamma:[0, \infty) \rightarrow[0, \infty)$ be a non-decreasing continuous function satisfying

$$
\begin{aligned}
\lim _{a \rightarrow \infty} \gamma(a) & =\infty, \\
\lim _{a \rightarrow \infty} \frac{m(\mathcal{E} \cap[0, a))}{\gamma(a)} & =\eta, \quad 0<\eta<\infty .
\end{aligned}
$$

Then, for each constant $C>0$, we have

$$
\begin{aligned}
\lim _{a \rightarrow \infty} \frac{m(\mathcal{E} \cap[0, C \gamma(a)))}{\gamma(a)} & =0, \\
\liminf _{a \rightarrow \infty} \frac{m(\mathcal{E} \cap[a-C \gamma(a), a))}{\gamma(a)} & =0 .
\end{aligned}
$$


Proof. It follows from (22) and (23) that

$$
\lim _{a \rightarrow \infty} \frac{m(\mathcal{E} \cap[0, C \gamma(a)))}{C \gamma(a)}=0 .
$$

Hence (25) holds.

In order to show (26), assume there exist $\delta>0$ and $a_{0}>0$ such that

$$
m(\mathcal{E} \cap[a-C \gamma(a), a))>\delta \gamma(a)>0 \quad \text { for } a \geq a_{0} .
$$

Equations (22) and (24) imply that $\lim _{a \rightarrow \infty}(a / \gamma(a))=\infty$. Therefore $\lim _{a \rightarrow \infty}(a-C \gamma(a))=\infty$. Since $\Gamma(a):=a-C \gamma(a)$ is a continuous function on $\mathbb{R}_{+}$with $\Gamma\left(a_{0}\right)<a_{0}$ and $\lim _{a \rightarrow \infty} \Gamma(a)=\infty$, there exists $a_{1}>a_{0}$ such that $\Gamma\left(a_{1}\right)=a_{1}-C \gamma\left(a_{1}\right)=a_{0}$. Hence, it follows from (27) that

$$
m\left(\mathcal{E} \cap\left[a_{0}, a_{1}\right)\right)>\delta \gamma\left(a_{1}\right)=\frac{\delta}{C}\left(a_{1}-a_{0}\right) .
$$

Continuing inductively in a similar way, we show that, for each $k \geq 1$, there exists $a_{k}>a_{k-1}$ such that $a_{k}-C \gamma\left(a_{k}\right)=a_{k-1}$, and consequently,

$$
m\left(\mathcal{E} \cap\left[a_{k-1}, a_{k}\right)\right)>\delta \gamma\left(a_{k}\right)=\frac{\delta}{C}\left(a_{k}-a_{k-1}\right), \quad k=1,2, \ldots
$$

(note that $a_{k} \rightarrow \infty$ as $k \rightarrow \infty$ since otherwise the left hand side of the inequality tends to 0 while the right hand side is always more than the positive number $\left.\delta \gamma\left(a_{0}\right)\right)$. If we sum up these inequalities with respect to $k$, we get

$$
m\left(\mathcal{E} \cap\left[a_{0}, a_{k}\right)\right)>\frac{\delta}{C}\left(a_{k}-a_{0}\right) .
$$

Hence

$$
\liminf _{k \rightarrow \infty} \frac{m\left(\mathcal{E} \cap\left[a_{0}, a_{k}\right)\right)}{a_{k}-a_{0}}=\liminf _{k \rightarrow \infty} \frac{m\left(\mathcal{E} \cap\left[0, a_{k}\right)\right)}{a_{k}}>\frac{\delta}{C}>0,
$$

which contradicts $(22)$.

Lemma 5. Let $\gamma$ and $\mathcal{E}$ be as in Lemma 4 and let, for $a>0, \bar{a}:=a / \gamma(a)$ and $\mathbb{1}_{\bar{a}}:=D_{\gamma(a)} \mathbb{1}_{\mathcal{E} \cap[0, a)}$ (see (15)). Then there exists a sequence $0<a_{n} \rightarrow$ $\infty$ such that

$$
H_{\left(0, \bar{a}_{n}\right)}\left(\mathbb{1}_{\bar{a}_{n}}\right)-H\left(\mathbb{1}_{\bar{a}_{n}}\right) \rightrightarrows 0 \quad \text { as } n \rightarrow \infty .
$$

Proof. It is sufficient to prove that for any $\varepsilon, \delta>0$ there exist $a>0$ such that

$$
m\left\{t \in \mathbb{R}:\left|H_{(0, \bar{a})}\left(\mathbb{1}_{\bar{a}}\right)(t)-H\left(\mathbb{1}_{\bar{a}}\right)(t)\right|>3 \varepsilon\right\}<4 \delta .
$$

Let

$$
C \geq \frac{4 \eta}{\pi \varepsilon}
$$


and take $a$ such that

$$
\begin{aligned}
\int_{0}^{\bar{a}} \mathbb{1}_{\bar{a}}(t) d t & =\frac{m(\mathcal{E} \cap[0, a))}{\gamma(a)}<2 \eta \\
\int_{0}^{C} \mathbb{1}_{\bar{a}}(t) d t & =\frac{m(\mathcal{E} \cap[0, C \gamma(a)))}{\gamma(a)}<\frac{\pi \varepsilon \delta}{2} \\
\int_{\bar{a}-C}^{\bar{a}} \mathbb{1}_{\bar{a}}(t) d t & =\frac{m(\mathcal{E} \cap[a-C \gamma(a), a))}{\gamma(a)}<\frac{\pi \varepsilon \delta}{2}
\end{aligned}
$$

Then, taking into account (31), (33) and (30), we have

$$
\begin{aligned}
0 \leq H\left(\mathbb{1}_{\bar{a}}\right)(\bar{a}+\delta) & =\frac{1}{\pi} \int_{0}^{\bar{a}} \mathbb{1}_{\bar{a}}(t) \frac{1}{\bar{a}+\delta-t} d t \\
& \leq \frac{1}{\pi C} \int_{0}^{\bar{a}-C} \mathbb{1}_{\bar{a}}(t) d t+\frac{1}{\pi \delta} \int_{a-C}^{\bar{a}} \mathbb{1}_{\bar{a}}(t) d t \\
& \leq \frac{1}{\pi C} \cdot 2 \eta+\frac{1}{\pi \delta} \cdot \frac{\pi \varepsilon \delta}{2} \leq \frac{\varepsilon}{2}+\frac{\varepsilon}{2}=\varepsilon
\end{aligned}
$$

In an analogous way, taking into account (31), (32) and (30), we get

$$
0 \geq H\left(\mathbb{1}_{\bar{a}}\right)(-\delta) \geq-\frac{1}{\pi \delta} \int_{0}^{C} \mathbb{1}_{\bar{a}}(t) d t-\frac{1}{\pi C} \int_{C}^{\bar{a}} \mathbb{1}_{\bar{a}}(t) d t \geq-\varepsilon .
$$

At the same time the function $H\left(\mathbb{1}_{\bar{a}}\right)$ is positive and decreasing on $(\bar{a}, \infty)$ and negative and decreasing on $(-\infty, 0)$. Hence

$$
\left|H\left(\mathbb{1}_{\bar{a}}\right)(t)\right| \leq \varepsilon \quad \text { for } t \in(-\infty,-\delta) \cup(\bar{a}+\delta, \infty) .
$$

It follows from (34) and Lemma 3 that

$$
\left|H_{(0, \bar{a})}\left(\mathbb{1}_{\bar{a}}\right)(t)-H\left(\mathbb{1}_{\bar{a}}\right)(t)\right| \leq 3 \varepsilon \quad \text { for } t \in(\delta, \bar{a}-\delta) .
$$

Relations (34) and (35) imply that

$$
\left\{t \in \mathbb{R}:\left|H_{(0, \bar{a})}\left(\mathbb{1}_{\bar{a}}\right)(t)-H\left(\mathbb{1}_{\bar{a}}\right)(t)\right|>3 \varepsilon\right\} \subset(-\delta, \delta) \cup(\bar{a}-\delta, \bar{a}+\delta)
$$

and (29) follows.

4. Proof of the Theorem. We consider the non-trivial case where $\mu(E) \neq 0$.

Obviously, it is enough to prove (2) for all $\lambda \geq 0$ except a countable number. Therefore, it can be assumed without loss of generality that $\lambda$ is a continuity point of the distribution function of $\mathbb{H}\left(\mathbb{1}_{E}\right)$. 
Let the functions $\left(\gamma_{x}\right)_{x \in X}$ be defined by (10). Fix any $x \in X$ which satisfies following conditions:

$$
\lim _{a \rightarrow \infty} \frac{1}{a} \int_{0}^{a} \mathbb{1}_{E}\left(T_{t} x\right) d t=0 \quad(\text { see }(12)) ;
$$

$\gamma(a):=\gamma_{x}(a)$ is a non-increasing continuous function satisfying (23);

$$
\lim _{a \rightarrow \infty} \frac{1}{\gamma(a)} \int_{0}^{a} \mathbb{1}_{E}\left(T_{t} x\right) d t=\mu(E) \quad(\text { see }(11)) ;
$$

the function $h(t):=\mathbb{1}_{E}\left(T_{t} x\right)$ is locally integrable on $\mathbb{R}$ and

$$
\lim _{a \rightarrow \infty} \frac{m\left\{0<t<a: H_{(0, a)}(h)(t)>\lambda\right\}}{\gamma(a)}=\mu\left\{\mathbb{H}\left(\mathbb{1}_{E}\right)>\lambda\right\}
$$

(see Lemma 2). If we set $\mathcal{E}=\left\{t \geq 0: T_{t} x \in E\right\}$, then (38) can be rewritten as

$$
\lim _{a \rightarrow \infty} \frac{m\left\{0<t<a: H_{(0, a)}\left(\mathbb{1}_{\mathcal{E} \cap[0, a)}\right)(t)>\lambda\right\}}{\gamma(a)}=\mu\left\{\mathbb{H}\left(\mathbb{1}_{E}\right)>\lambda\right\}
$$

and $m(\mathcal{E} \cap[0, a))=\int_{0}^{a} \mathbb{1}_{E}\left(T_{t} x\right) d t$. It follows respectively from (36) and (37) that (22) and (24) hold. Thus, we can apply Lemma 5 to conclude that there exists a sequence of positive numbers $a_{n} \rightarrow \infty$ such that (28) holds.

Since $\mathbb{1}_{\bar{a}_{n}}=D_{\gamma\left(a_{n}\right)} \mathbb{1}_{\mathcal{E} \cap\left[0, a_{n}\right)}$ is the characteristic function of the measurable set $\left\{t \geq 0: \gamma\left(a_{n}\right) t \in \mathcal{E} \cap\left[0, a_{n}\right)\right\}$ (see Lemma 5) with measure $\left(1 / \gamma\left(a_{n}\right)\right) m\left(\mathcal{E} \cap\left[0, a_{n}\right)\right)$, by the Stein-Weiss Theorem A we have

$$
m\left\{H\left(\mathbb{1}_{\bar{a}_{n}}\right)>\lambda\right\}=\Psi\left(\frac{1}{\gamma\left(a_{n}\right)} m\left(\mathcal{E} \cap\left[0, a_{n}\right)\right), \lambda\right), \quad n=1,2, \ldots
$$

Hence $\lim _{n \rightarrow \infty} m\left\{H\left(\mathbb{1}_{\bar{a}_{n}}\right)>\lambda\right\}=\Psi(\mu(E), \lambda)$ since $\Psi$ is a continuous function and $\lim _{a \rightarrow \infty}(1 / \gamma(a)) m(\mathcal{E} \cap[0, a))=\mu(E)$ (see $\left.(37)\right)$.

We can now use Lemma 1 , where $F_{n}=H_{\left(0, \bar{a}_{n}\right)}\left(\mathbb{1}_{\bar{a}_{n}}\right), G_{n}=H\left(\mathbb{1}_{\bar{a}_{n}}\right)$ and $\psi(\lambda)=\Psi(\mu(E), \lambda)$, to conclude that

$$
\lim _{n \rightarrow \infty} m\left\{H_{\left(0, \bar{a}_{n}\right)}\left(\mathbb{1}_{\bar{a}_{n}}\right)>\lambda\right\}=\Psi(\mu(E), \lambda) .
$$

According to (16) we have

$$
m\left\{H_{\left(0, \bar{a}_{n}\right)}\left(\mathbb{1}_{\bar{a}_{n}}\right)>\lambda\right\}=\frac{1}{\gamma\left(a_{n}\right)} m\left\{H_{\left(0, a_{n}\right)}\left(\mathbb{1}_{\mathcal{E} \cap\left[0, a_{n}\right)}\right)>\lambda\right\}
$$

and, taking into account (39), we get

$$
\lim _{n \rightarrow \infty} m\left\{H_{\left(0, \bar{a}_{n}\right)}\left(\mathbb{1}_{\bar{a}_{n}}\right)>\lambda\right\}=\mu\left\{\mathbb{H}\left(\mathbb{1}_{E}\right)>\lambda\right\} .
$$

Equalities (40) and (41) imply the first equality in (2). The second can be proved in the same manner. 


\section{References}

[1] A. P. Calderón, Singular integrals, Bull. Amer. Math. Soc. 72 (1966), 427-465.

[2] A. P. Calderón and A. Zygmund, Singular integrals and periodic functions, Studia Math. 14 (1954), 249-271.

[3] L. Ephremidze, On the generalization of the Stein-Weiss theorem for the ergodic Hilbert transform, ibid. 155 (2003), 67-75.

[4] J. B. Garnett, Bounded Analytic Functions, Academic Press, New York, 1981.

[5] Sh. Hasegava and R. Sato, A general ratio ergodic theorem for semigroups, Pacific J. Math. 62 (1976), 435-437.

[6] K. E. Petersen, Another proof of the existence of the ergodic Hilbert transform, Proc. Amer. Math. Soc. 88 (1983), 39-43.

[7] K. E. Petersen, Ergodic Theory, Cambridge Univ. Press, Cambridge, 1983.

[8] E. M. Stein and G. Weiss, An extension of a theorem of Marcinkiewicz and some of its applications, J. Math. Mech. 8 (1958), 263-284.

[9] O. D. Tsereteli, Metric properties of conjugate functions, Itogi Nauki i Tekhniki 7 (1975), 18-57 (in Russian).

A. Razmadze Mathematical Institute

1, M. Aleksidze Str.

380093 Tbilisi, Georgia

E-mail: lasha@rmi.acnet.ge

Received July 7, 2003

Revised version January 19, 2004 